\#28613

\title{
A 96-well Plate Assay for CYP3A Induction Using Cryopreserved Human Hepatocytes
}

Naomi Kamiguchi, Eiji Aoyama, Teruaki Okuda, and Toshiya Moriwaki

Discovery Research Center, Pharmaceutical Research Division, Takeda Pharmaceutical Company

Limited (N.M., T.O., T.M.); Development Research Center, Pharmaceutical Research Division,

Takeda Pharmaceutical Company Limited (E.A.) 
Running title: A 96-well plate assay for CYP3A induction

Corresponding author: Naomi Kamiguchi

Discovery Research Center, Pharmaceutical Research Division

Takeda Pharmaceutical Company Limited

2-17-85 Juso-Honmachi, Yodogawa-ku, Osaka 532-8686, Japan

Phone: (+81) 6-6308-9085

Fax: (+81) 6-6308-9019

Number of text pages: 18

Number of tables: 1

Number of figures: 5

Number of references: 19

Number of words in the Abstract: 250

Number of words in the Introduction: 505

Number of words in the Results and Discussion: 1197

\begin{abstract}
Abbreviations:
CYP, cytochrome P450; TL, Thin-layer; DDIs, drug-drug interactions; NCEs, new chemical entities; ; qRT-PCR, quantitative reverse transcriptase polymerase chain reaction; PLO/LM, poly-L-ornithine/laminin; PDL, poly-D-lysine; RIF, rifampicin; UPLC, ultra-high performance liquid chromatography; DMSO, dimethyl sulfoxide; HEPES, 2-[4-(2-Hydroxyethyl)-1-piperazinyl] ethanesulfonic acid.
\end{abstract}




\section{Abstract}

A reliable and practical cytochrome P450 (CYP) 3A induction assay with cryopreserved human hepatocytes in a 96-well format was developed. Various 96-well plates with different basement membrane were evaluated using prototypical inducers, rifampicin, phenytoin, and carbamazepin. Thin-layer (TL) matrigel was found to yield the highest basal and induced levels of CYP3A activity as determined by testosterone 6 $\beta$-hydroxylation. Concentration-dependent CYP3A induction of rifampicin was reproducible with the $\mathrm{EC}_{50}$ values of $0.36 \pm 0.28 \mu \mathrm{M}$ from four batches of human hepatocytes using the 96-well plate with TL matrigel. The rank order of induction potency for 9 inducers or non-inducers at a concentration of $10 \mu \mathrm{M}$ were well comparable among the multiple donors, by expressing the results as percentage of change compared with the positive control, 10 $\mu \mathrm{M}$ rifampicin. Co-treatment of avasimibe or efavirenz with $10 \mu \mathrm{M}$ rifampicin was found to reduce CYP3A activities induced by rifampicin lower than that treated rifampicin alone, whereas the phenobarbital and carbamazepine had no effect. From a comparison of induced CYP3A activities and gene expression levels, there were compounds that would cause induction of CYP3A4 mRNA but not activity, presumably due to their inhibitory effect on CYP3A activity. The co-treatment assay of test compound with rifampicin allows us to exclude the false negative results caused by the cytotoxicity and/or the mechanism-based inactivation, when the drug candidate's ability for CYP3A induction is evaluating by the enzyme activity. This 96-well plate assay, which is robust, reproducible and convenient, has demonstrated the paramount applicability to the early drug discovery stage. 


\section{Introduction}

Cytochrome P450 (CYP) 3A is regarded as one of the predominant CYP isoforms among drug metabolizing enzymes expressed in the human liver. The induction of CYP3A can result in clinically significant drug-drug interactions (DDIs) or autoinductions. The CYP3A inducers may cause reduction in therapeutic efficacy of comedication, or an increase in risk of metabolite-induced toxicity. Therefore, it is important to evaluate the induction potential of new chemical entities (NCEs) in the early discovery stage (Dickins, 2004; Luo et al., 2004; Lin, 2006).

Several in vitro models have been developed to assess the potential of CYP3A induction, including nuclear receptor-based assays (Moore et al., 2000; El Sankary et al., 2001; Luo et al., 2002; Sinz et al., 2006), immortalized cell lines (Mills et al., 2004; Hariparsad et al., 2008), and primary hepatocytes. Among these models, the primary human hepatocytes have been considered as the most predictive model for assessing in vitro induction of CYP enzymes. Although fresh human hepatocytes are the standard for evaluating induction potential, attachable cryopreserved hepatocytes have been used more often due to increased availability. The enzymes in cryopreserved hepatocytes were shown to be inducible by standard CYP inducers with the increased levels of mRNA, protein and/or functional enzyme activity as the endpoint (Silva et al., 1999; Roymans et al., 2004, 2005).

A high-speed screening method for the evaluation of CYP1A2 and CYP3A4 induction potency has been established using real-time one-step RT-PCR in cryopreserved human hepatocytes (Nishimura et al., 2009). However, there have been few reports on the CYP induction assay procedures using cryopreserved hepatocytes with catalytic activity as the endpoint in microplates with 96 or more wells per plates yet. Although mRNA expression levels are also used for evaluating CYP induction, measurement of the enzyme activities has been recommended by FDA (2006) as the most reliable method for quantifying a drug's induction potential. Moreover, extraction of total RNA from hepatocytes and the measurement of the mRNA levels in addition to the determination of 
CYP activities is a time and cost consuming process of CYP induction screening in the early discovery stage.

Fahmi et al. have recently demonstrated the applicability of a relative induction score (RIS) to predict clinical DDI using the human cryopreserved hepatocytes model (2009). The RIS approach was based on combining in vitro induction parameters $\left(\mathrm{EC}_{50}\right.$ and $\left.\mathrm{E}_{\max }\right)$ from CYP3A4 mRNA induction data with the efficacious free plasma concentrations, and the RIS values were well correlated to the magnitude of clinical DDIs. However, donor-to-donor variability in the human hepatocytes has remained unresolved, in which in vitro induction parameters varied considerably among different batches of hepatocytes from different donors.

The aim of this study was to develop a reliable CYP3A induction assay method for drug discovery using cryopreserved human hepatocytes with CYP3A activity as the endpoint. We show that thin-layer (TL) matrigel as the basement membrane on the bottom of 96-well plates is useful for maintaining the steady expression of CYP3A activity in cryopreserved hepatocytes. This CYP3A induction method with TL matrigel was characterized using four batches of cryopreserved human hepatocytes. 


\section{Materials and Methods}

Materials. Cryopreserved human hepatocytes from four donors were obtained from Celsis In Vitro Technologies (lot LOF, UFN, and LMP; Baltimore, MD) and CellzDirect, Inc. (lot 4201; Pittsboro, NC). Hepatocyte plating medium (In VitroGRO CP Medium), incubation medium (In VitroGRO HI Medium) and Torpedo Antibiotic Mix were obtained from Celsis In Vitro Technologies. Multi-well plates coated with thin-layer (TL) matrigel, collagen type I, collagen type IV, laminin, fibronectin, poly-L-ornithine/laminin (PLO/LM) and poly-D-lysine (PDL) were obtained from BD Biosciences (San Jose, CA). Rifampicin, phenytoin, carbamazepine, nifedipine, sulfinpyrazone, cimetidine, furosemide, and Phenobarbital sodium were purchased from WAKO Pure Chemical Industries, Ltd. (Osaka, Japan). Modafinil was purchased from Sigma-Aldrich (St. Louis, MO). Efavirenz, avasimibe, and ritonavir were produced at Takeda Pharmaceutical Company Limited (Osaka, Japan). All other chemicals were of the highest grade commercially available.

Cell culturing and induction. Cryopreserved human hepatocytes were thawed in plating medium and transferred to TL matrigel 96-well plates at the density of $0.4-0.8 \times 10^{5}$ viable cells/well. After $24 \mathrm{~h}$ incubation, the plating medium was removed and replaced with incubation medium supplemented with Torpedo Antibiotic Mix. The cells were then incubated for 3 additional days to establish the cultures. Following the initial 4-day conditioning culture, the medium was removed and the hepatocytes were treated daily for 3 days with incubation medium including test compounds in the absence or presence of $10 \mu \mathrm{M}$ rifampicin. The wells of test compounds with 10 $\mu \mathrm{M}$ rifampicin were used to estimate the inhibitory effect of test compound on rifampicin-induced (RIF-induced) CYP3A activity. All incubations were conducted in a humidified atmosphere at $37^{\circ} \mathrm{C}$ in $5 \% \mathrm{CO}_{2}$.

CYP3A activity measurement. CYP3A activity was quantified by measuring the extent of $6 \beta$-hydroxy testosterone formation from testosterone by ultra-performance liquid chromatography systems (UPLC; Waters, Milford, MA). The cultured hepatocytes were washed 2 times with Hanks' 
balanced salt solution with $25 \mathrm{mM}$ HEPES, and incubated with $150 \mu \mathrm{L}$ of the same buffer containing $100 \mu \mathrm{M}$ testosterone at $37^{\circ} \mathrm{C}$ for 2 hours. At the end of the incubation, $120 \mu \mathrm{L}$ aliquot of the media was transferred to a 96-well plate containing $10 \mu \mathrm{L} 5 \% \mathrm{HClO}_{4}$, and $10 \mu \mathrm{L}$ of the mixture was injected onto the column. Testosterone and $6 \beta$-hydroxy testosterone were chromatographed using an Acquity BEH C18 column $(100 \times 2.1 \mathrm{~mm}, 1.7 \mu \mathrm{m}$; Waters $)$ and UV detection at $250 \mathrm{~nm}$. The mobile phase consist of solvent A (10 mM ammonium acetate/water/acetonitrile, 1:8:1) and solvent B (10 mM ammonium acetate/acetonitrile, 1:9), with a gradient profile starting at $25 \% \mathrm{~B}$ and then increasing to $95 \% \mathrm{~B}$ from $1 \mathrm{~min}$ to $1.01 \mathrm{~min}$, and remaining at $95 \% \mathrm{~B}$ until $2.4 \mathrm{~min}$, then returning back to $25 \% \mathrm{~B}$ at $2.41 \mathrm{~min}$ followed by re-equilibration until $2.5 \mathrm{~min}$, all at flow rate of 0.5 $\mathrm{mL} / \mathrm{min}$.

Real-Time qRT-PCR. Total RNA was isolated using the SV 96 Total RNA Isolation Kit (Promega, Madison, WI). Quantification of cytochrome CYP3A4 mRNA was performed in a 384-well format using the TaqMan One-Step reverse transcription-polymerase chain reaction method by using the ABI 7900HT sequence detection system (Applied Biosystems, Foster City, CA). The relative quantity of the target CYP3A4 gene compared with the endogenous control (Hypoxanthine phosphoribosyltransferase I) was determined by the $\Delta \Delta \mathrm{CT}$ method.

Data analysis. The values of percentage of rifampicin response (\% RIF) and fold of control (Fold) were calculated as below:

$$
\begin{aligned}
& \% \mathrm{RIF}=\frac{\text { activity of test compound treated cells }- \text { activity of control }}{\text { activity of } 10 \mu \mathrm{M} \text { rifampicin }- \text { activity of control }} \times 100 \\
& \text { Fold }=\frac{\text { activity of test compound treated cells }}{\text { activity of control }}
\end{aligned}
$$

Standard deviations of Fold and \%RIF were calculated using the 'propagation of error' formula.

To determine the $\mathrm{EC}_{50}$ and $\mathrm{E}_{\max }$ values, the data from concentration response curves were fitted to a three parameter sigmoid model. All curve fitting were carried out with GraphPad Prism 5.0 
\#28613

(GraphPad Software, La Jolla, CA ). 


\section{Results and Discussion}

\section{Assessment of various plates with different attachment substratum on CYP3A induction}

response. Because long-term culture ( 8 days) is required for CYP induction assays, the improvement of cell attachment and prevention of gradual decline of CYP3A activity plays an important role in the assay construction. We first assessed the 96-well plates with various types of basement membrane on the bottom of the plates. CYP3A inducibilities of human hepatocytes cultured in 96-well plates were compared for the seven types of basement membrane, including extracellular matrix components or synthetic compounds to enhance cell attachment, using prototypical inducers, rifampicin, phenytoin, and carbamazepin. Hepatocytes plated in 96-well plates with each basement membrane were allowed to recovery the culture for 3 days in incubation medium, and then incubated for additional 3 days with inducers as described under Materials and Methods. TL matrigel plates yielded the higher basal and induced CYP3A activity among these tested plates (Table 1). When the CYP3A induction potential were expressed as change in percentage compared with $10 \mu \mathrm{M}$ rifampicin (\% RIF), the positive control, the CYP3A induction by phenytoin and carbamazepine in the TL matrigel plates were significantly higher than in the other plates. Although the most common culture format is collagen type I and collagen-matrigel sandwich culture (Chu et al., 2009), there are reports that matrigel overlay to cultured primary rat hepatocytes had enhanced phenobarbital responsiveness on CYP2B and CYP3A gene induction (Sidhu et al., 1993, 2004). We observed the higher \% RIF values of the CYP3A induction by carbamazepine and phenytoin in the TL matrigel culture, this finding suggest that matrigel may enhance the CYP3A inducibility by unclear mechanism, not only improve cell attachment onto the 96-well plate. In addition, the TL matrigel plate coated on the bottom of the plate is easier to handle than gelled matrigel plate commonly used as three-dimensional culture. From the results of this study, we have employed the TL matrigel plate for our CYP3A induction assay system in a 96-well format.

Assay Characterization with TL matrigel 96-well plates. To validate TL matrigel plate for 
CYP3A induction assay, we evaluated prototypical inducers using the TL matrigel 96-well plate in different batches of human hepatocytes. Concentration-response studies with rifampicin were first conducted in four batches of human hepatocytes. As shown in Fig. 1, rifampicin-mediated CYP3A induction was reproducible in individual donors of hepatocytes, with the $\mathrm{EC}_{50}$ values of $0.36 \pm 0.28$ $\mu \mathrm{M}$. Whereas the magnitude of CYP3A induction response expressed as fold increase varied according to the batches (data not shown), the variation among batches was reduced by using $\%$ RIF as an indicator of CYP3A induction response. To assess the ability and reproducibility of the assay, 6 inducers and 2 non-inducers were evaluated with this established method in four batches of human hepatocytes. The compounds used as inducers were rifampicin, efavirenz, nifedipine, phenytoin, carbamazepine, sulfinpyrazone, and modafinil. The compounds used as non-inducers were cimetidine and furosemide. All compounds were tested at $10 \mu \mathrm{M}$. As a result, CYP3A induction responses of the tested compounds expressed as \% RIF were well comparable in different donors of hepatocytes, and. the rank order of inducers and non-inducers was reproducible in this assay (Fig. 2). These results indicate that this established CYP3A induction assay method with TL matrigel plate is robust and reliable for evaluating the ranking of CYP3A induction potency for candidate compounds.

Co-treatment assay of prototypical inducers with rifampicin. The bell-shaped response of CYP3A induction is commonly encountered, in which the induction response decreased at higher concentrations of inducers. For a screening assay using only one or two test concentration points, the phenomenon of bell-shaped response is considered to be a risk of false-negative resuls in evaluating the CYP3A induction potency. Cytotoxicity, mechanism-based CYP3A inactivity, and/or solubility are often used to explain the bell-shaped response. Whereas commonly assessment of the cytotoxicity during the course of an experiment is recommended (Chu et al., 2009), it is insufficient to explain the other causes of bell-shaped response. Therefore, we constructed the co-treatment assay of rifampicin with a test compound to detect the compound property to exhibit the bell-shaped 
CYP3A induction response. Fig. 3 shows the CYP3A induction by prototypical inducers, phenobarbital, carbamazepine, avasimibe, and efavirenz, in which hepatocytes were treated with the inducer alone, or with the inducer and rifampicin. In this assay, hepatocytes were incubated with the inducer and $10 \mu \mathrm{M}$ rifampicin for 3 days, and the induced CYP3A activities were determined. Phenobarbital and carbamazepine increased the CYP3A activity in a concentration dependent manner. The rifampicin-induced (RIF-induced) CYP3A activities were constant in the co-treatment with phenobarbital or carbamazepine, even at the higher concentration of these inducers (Fig. 3). On the other hand, avasimibe and efavirenz showed the bell-shaped response with decreasing in the induced CYP3A activity at higher concentrations (Fig. 3). In the co-treatment of avasimibe or efavirenz, the RIF-induced CYP3A activity decreased with increasing the concentrations of avasimibe or efavirenz. These results indicate that avasimibe and efavirenz have inhibitory effects on the RIF-induced CYP3A activity for some reasons, i.e. cytotoxicity or mechanism-based CYP3A inactivation. By implementing the CYP3A induction assay together with the co-treatment assay, we can exclude the false negative results in selecting drug candidates with no CYP3A induction potency, while the causes of the inhibitory effects of the compounds are unidentified in this co-treatment assay.

Comparison of induced CYP3A activities and mRNA levels. Ritonavir, mechanism-based inhibitor as well as inducer of CYP3A, indicated the inhibitory effect on the RIF-induced CYP3A activity in the co-treatment assay (Fig.4). On the other hand, ritonavir was found not to inhibit the induction of CYP3A4 mRNA of rifampicin in same hepatocytes. In the case that mechanism-based inactivation is a cause of inhibitory effect on CYP3A induction, measuring CYP3A4 mRNA expression could provide the useful information to evaluate whether the compounds has the induction potential or not. Fig. 5 shows a correlation between CYP3A activities and CYP3A4 mRNA levels with 44 compounds at various concentrations. The induced CYP3A activity and mRNA level by tests compounds alone were used for the plots on the graph, in which the 
compounds were categorized by the results of the co-treatment assay according to the potency of inhibitory effect on CYP3A induction by rifampicin. The CYP3A activity and CYP3A4 mRNA levels induced by the test compounds were well correlated, when the compounds were weak or no inhibitor on RIF-induced CYP3A activity, in which residual percentage of the RIF-induced CYP3A activity was more than $80 \%$. The compounds that would cause induction of CYP3A4 mRNA expression but not CYP3A activity were found to have potent inhibitory effect on the RIF-induced CYP3A activity. These compounds suppressed the RIF-induced CYP3A activity less than 30\% compared with those induced rifampicin alone. The results suggest that the measuring CYP3A4 mRNA is effective to assess intrinsic CYP3A induction potential especially in the case that the test compounds have inhibitory effect on the RIF-induced CYP3A activity.

In conclusion, we established a new CYP3A induction assay system using cryopreserved hepatocytes in a 96-well format with TL matrigel plate. Our assay system offers several advantages of higher-capacity, robustness, and reproducibility. This assay system is applicable to the early drug discovery stage to rank the CYP3A induction potential for a large number of new drug candidates. 


\section{References}

Chu V, Einolf HJ, Evers R, Kumar G, Moore D, Ripp S, Silva J, Sinha V, Sinz M, and Skerjanec A (2009) In vitro and in vivo induction of cytochrome p450: a survey of the current practices and recommendations: a pharmaceutical research and manufacturers of america perspective. Drug Metab Dispos 37: 1339-1354.

Dickins M (2004) Induction of cytochromes P450. Curr Top Med Chem 4: 1745-1766.

El Sankary W, Gibson GG, Ayrton A, and Plant N (2001) Use of a reporter gene assay to predict and rank the potency and efficacy of CYP3A4 inducers. Drug Metab Dispos 29: 1499-1504.

Fahmi OA, Boldt S, Kish M, Obach RS and Tremaine LM (2008) Prediction of drug-drug interactions from in vitro induction data: application of the relative induction score approach using cryopreserved human hepatocytes. Drug Metab Dispos. 36:1971-1974.

FDA (2006) Guidance for industry - Drug Interaction Studies - Study Design, Data Analysis, and Implications for Dosing and Labeling

Hariparsad N, Carr BA, Evers R, and Chu X (2008) Comparison of immortalized Fa2N-4 cells and human hepatocytes as in vitro models for cytochrome P450 induction. Drug Metab Dispos 36: 1046-1055.

Lin JH (2006) CYP induction-mediated drug interactions: in vitro assessment and clinical implications. Pharm Res 23: 1089-1116.

Luo G, Cunningham M, Kim S, Burn T, Lin J, Sinz M, Hamilton G, Rizzo C, Jolley S, Gilbert D, Downey A, Mudra D, Graham R, Carroll K, Xie J, Madan A, Parkinson A, Christ D, Selling B, LeCluyse E, and Gan LS (2002) CYP3A4 induction by drugs: correlation between a pregnane X receptor reporter gene assay and CYP3A4 expression in human hepatocytes. Drug Metab Dispos 30: 795-804.

Luo G, Guenthner T, Gan LS, and Humphreys WG (2004) CYP3A4 induction by xenobiotics: biochemistry, experimental methods and impact on drug discovery and development. Curr Drug 
Metab 5: 483-505.

Mills JB, Rose KA, Sadagopan N, Sahi J, and de Morais SM (2004) Induction of drug metabolism enzymes and MDR1 using a novel human hepatocyte cell line. J Pharmacol Exp Ther 309: 303-309.

Moore JT, and Kliewer SA (2000) Use of the nuclear receptor PXR to predict drug interactions. Toxicology 153: 1-10.

Nishimura M, Narimatsu S, and Naito S (2009) Evaluation of induction potency of new drug candidates on CYP1A2 and CYP3A4 using real-time one-step RT-PCR in primary cultures of cryopreserved human hepatocytes. Drug Metab Pharmacokinet. 24:446-450.

Ripp SL, Mills JB, Fahmi OA, Trevena KA, Liras JL, Maurer TS, and de Morais SM (2006) Use of immortalized human hepatocytes to predict the magnitude of clinical drug-drug interactions caused by CYP3A4 induction. Drug Metab Dispos 34: 1742-1748.

Roymans D, Van Looveren C, Leone A, Parker JB, McMillian M, Johnson MD, Koganti A, Gilissen R, Silber P, Mannens G, and Meuldermans W (2004) Determination of cytochrome P450 1A2 and cytochrome P450 3A4 induction in cryopreserved human hepatocytes. Biochem Pharmacol 67: 427-437.

Roymans D, Annaert P, Van Houdt J, Weygers A, Noukens J, Sensenhauser C, Silva J, Van Looveren C, Hendrickx J, Mannens G, and Meuldermans W (2005) Expression and induction potential of cytochromes P450 in human cryopreserved hepatocytes. Drug Metab Dispos 33: 1004-1016.

Sidhu JS, Farin FM, and Omiecinski CJ (1993) Influence of extracellular matrix overlay on phenobarbital-mediated induction of CYP2B1, 2B2, and 3A1 genes in primary adult rat hepatocyte culture. Arch Biochem Biophys 301: 103-113.

Sidhu JS, Liu F, and Omiecinski CJ (2004) Phenobarbital responsiveness as a uniquely sensitive indicator of hepatocyte differentiation status: requirement of dexamethasone and extracellular 
matrix in establishing the functional integrity of cultured primary rat hepatocytes. Exp Cell Res 292: $252-264$.

Silva JM, Day SH, and Nicoll Griffith DA (1999) Induction of cytochrome-P450 in cryopreserved rat and human hepatocytes. Chem Biol Interact 121: 49-63.

Sinz M, Kim S, Zhu Z, Chen T, Anthony M, Dickinson K, and Rodrigues AD (2006) Evaluation of 170 xenobiotics as transactivators of human pregnane $\mathrm{X}$ receptor (hPXR) and correlation to known CYP3A4 drug interactions. Curr Drug Metab 7: 375-388. 


\section{Legends for Figures}

Fig. 1. Concentration-response curve for CYP3A induction by rifampicin in cryopreserved human hepatocytes from four different donors. Data represent mean \pm standard deviation calculated from four separate wells. Data points were fitted to a three-parameter sigmoid model.

Fig. 2. CYP3A induction in cryopreserved human hepatocytes from four different donors treated with various inducers (rifampicin, efavirenz, nifedipine, phenytoin, carbamazepine, sulfinpyrazone, or modafinil) and non-inducers (cimetidine or furosemide). The CYP3A induction response was expressed as a percentage of the $10 \mu \mathrm{M}$ rifampicin response calculated from four separate wells. Error bars indicate standard deviation.

Fig. 3. Concentration-response curves for CYP3A induction by phenobarbital (A), carbamazepine (B), avasimibe(C), and efavirenz (D). Hepatocytes (lot LOF) were treated for 3 days with the inducer alone (closed circle) or in combination with $10 \mu \mathrm{M}$ rifampicin (open circle). Data are expressed as the mean \pm S.D. of CYP3A activity ( $n=3$ or 4$)$.

Fig. 4. Concentration-response curves for induction of CYP3A activity (A) and CYP3A4 mRNA expression (B) by ritonavir. Hepatocytes (lot LOF) were treated with ritonavir alone (closed circle) or in combination with $10 \mu \mathrm{M}$ rifampicin (open circle). Data are expressed as the mean \pm S.D. of CYP3A activity or the mean of CYP3A4 mRNA level ( $n=3$ or 4$)$.

Fig. 5. Correlation between induced CYP3A activity and CYP3A4 mRNA level. Cryopreserved hepatocytes (lot LOF, UFN) were treated with 44 compounds at several concentrations $(n=128)$. The induced CYP3A activity and mRNA level by tests compounds alone were used for the plots. The data points were categorized by magnitude of the inhibitory effect of test compounds on 
RIF-induced CYP3A induction into three classes, > 80\% (open circle, non/weak), 30 - 80\% (closed triangle, middle), and $<30 \%$ (closed circle, potent), according to the percentages of the remaining CYP3A activity treated with $10 \mu \mathrm{M}$ rifampicin in the presence of test compound to the activity treated with rifampicin alone. Data represent mean calculated from four separate wells. 
Table 1. Comparison of 96-well plates with various types of basement membrane on CYP3A induction. Hepatocytes (lot.LOF) were cultured on a 96-well plate coated with 2 lots of thin-layer matrigel, collagen type I, collagen type IV, laminin, fibronectin, poly-L-ornithine/laminin (PLO/LM), or poly-D-lysine (PDL). Hepatocytes cultures were treated with $0.1 \%$ DMSO (control), $10 \mu \mathrm{M}$ rifampicin, $30 \mu \mathrm{M}$ phenytoin, or $30 \mu \mathrm{M}$ carbamazepine, and determined the induced CYP3A activity. CYP3A induction responses determined by $6 \beta$-hydroxytestosterone formation are represented as CYP3A activity (pmol/h/million cells), fold induction, or \% RIF. Data represent mean \pm standard deviation calculated from four separate wells.

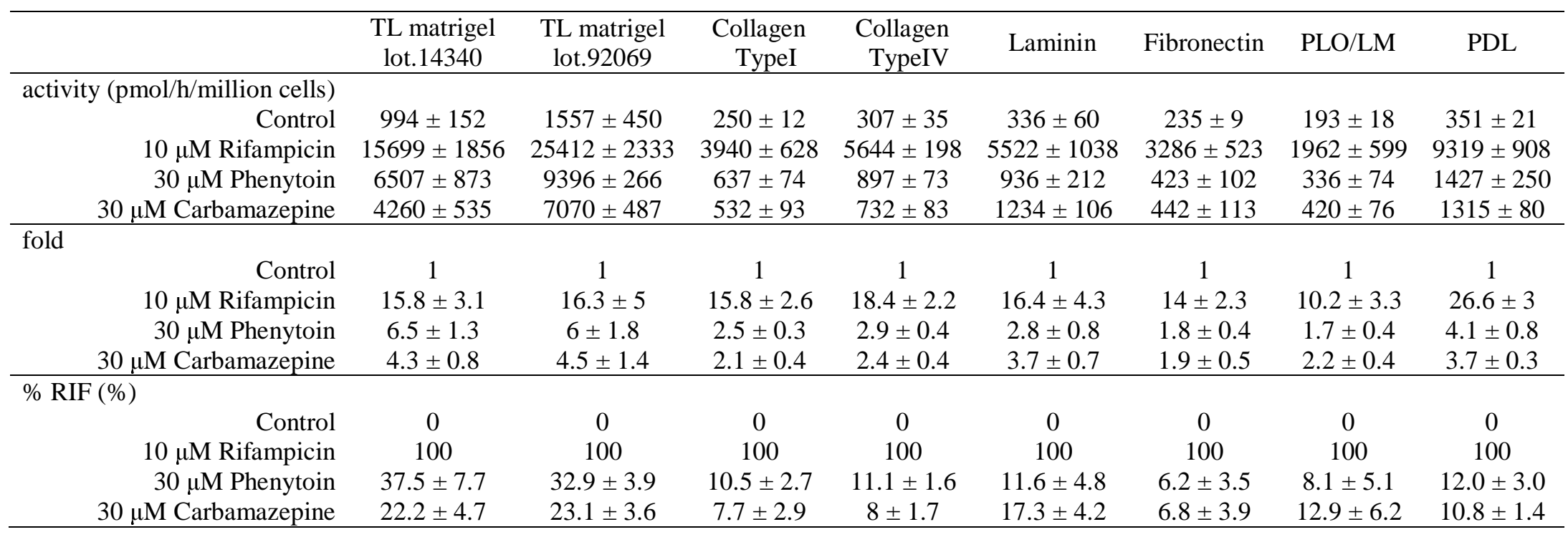


Figure 1

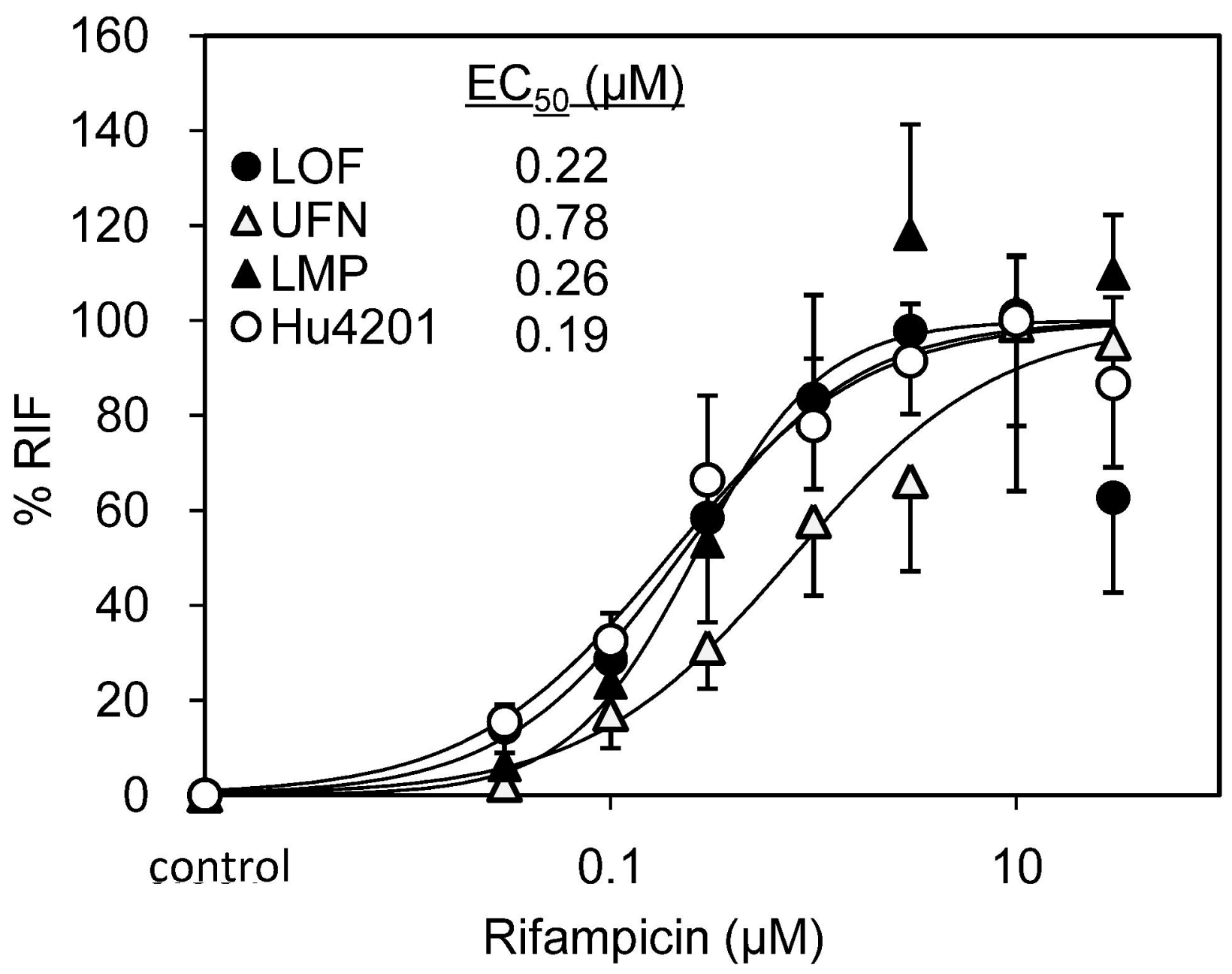


Figure 2

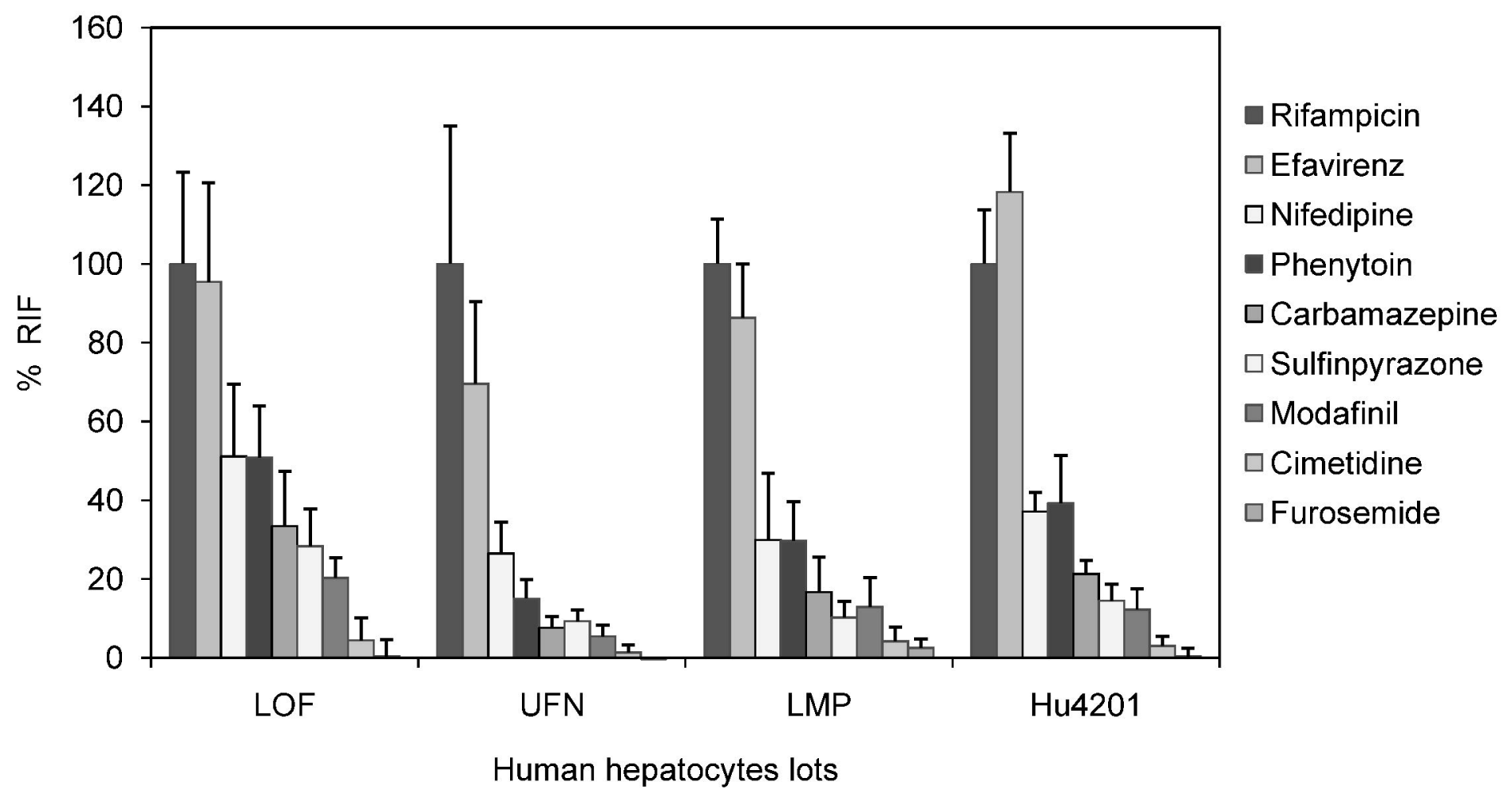


Figure 3

DMD Fast Forward. Published on August 10, 2010 as DOI: 10.1124/dmd.109.028613

This article has not been copyedited and formatted. The final version may differ from this version.
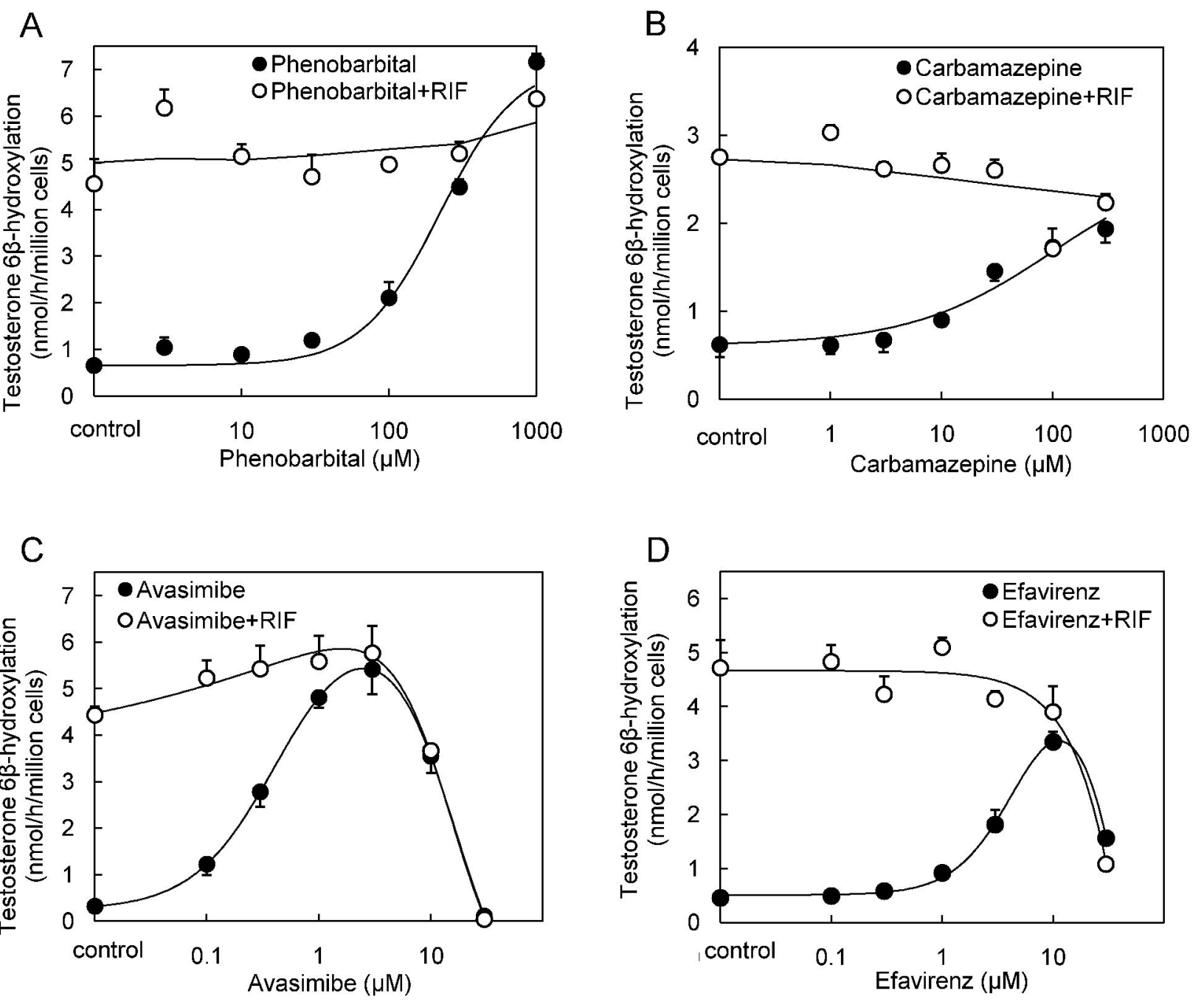
Figure 4

DMD Fast Forward. Published on August 10, 2010 as DOI: 10.1124/dmd.109.028613

This article has not been copyedited and formatted. The final version may differ from this version.
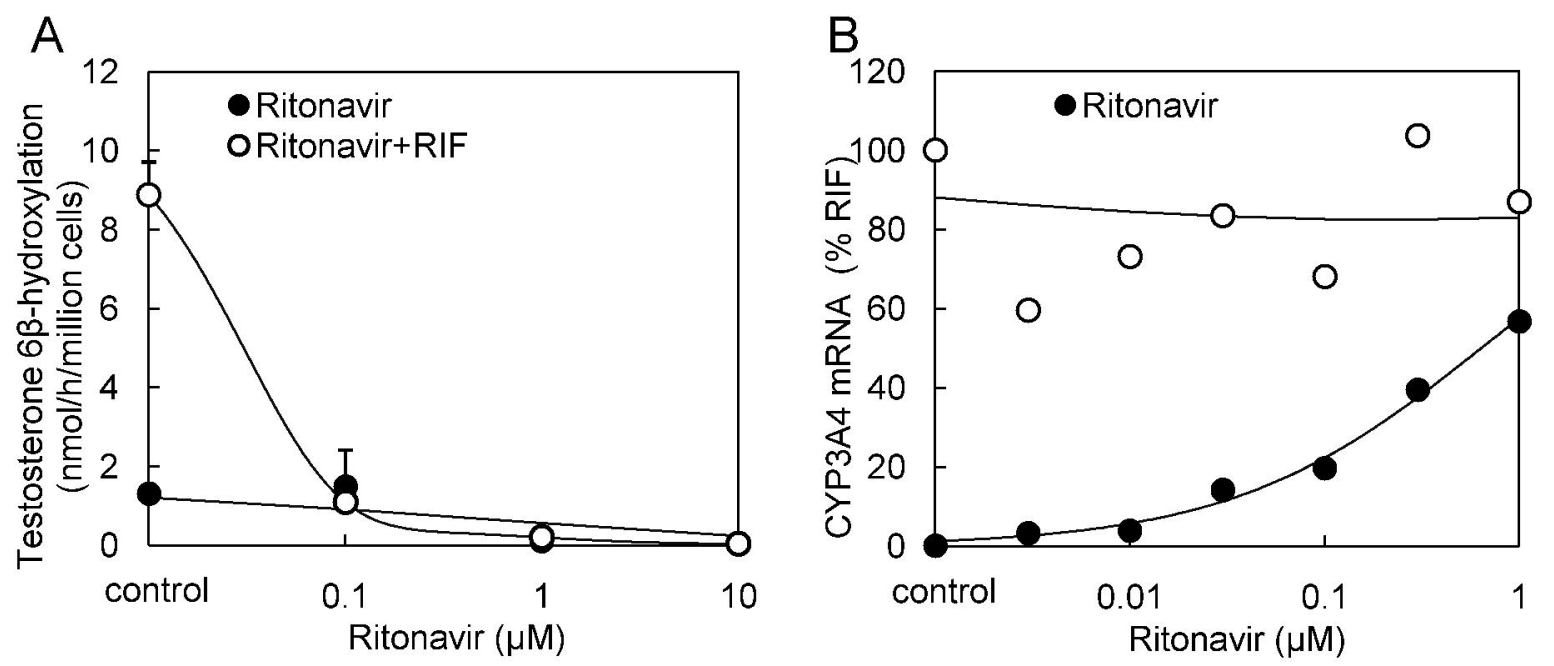
Figure 5

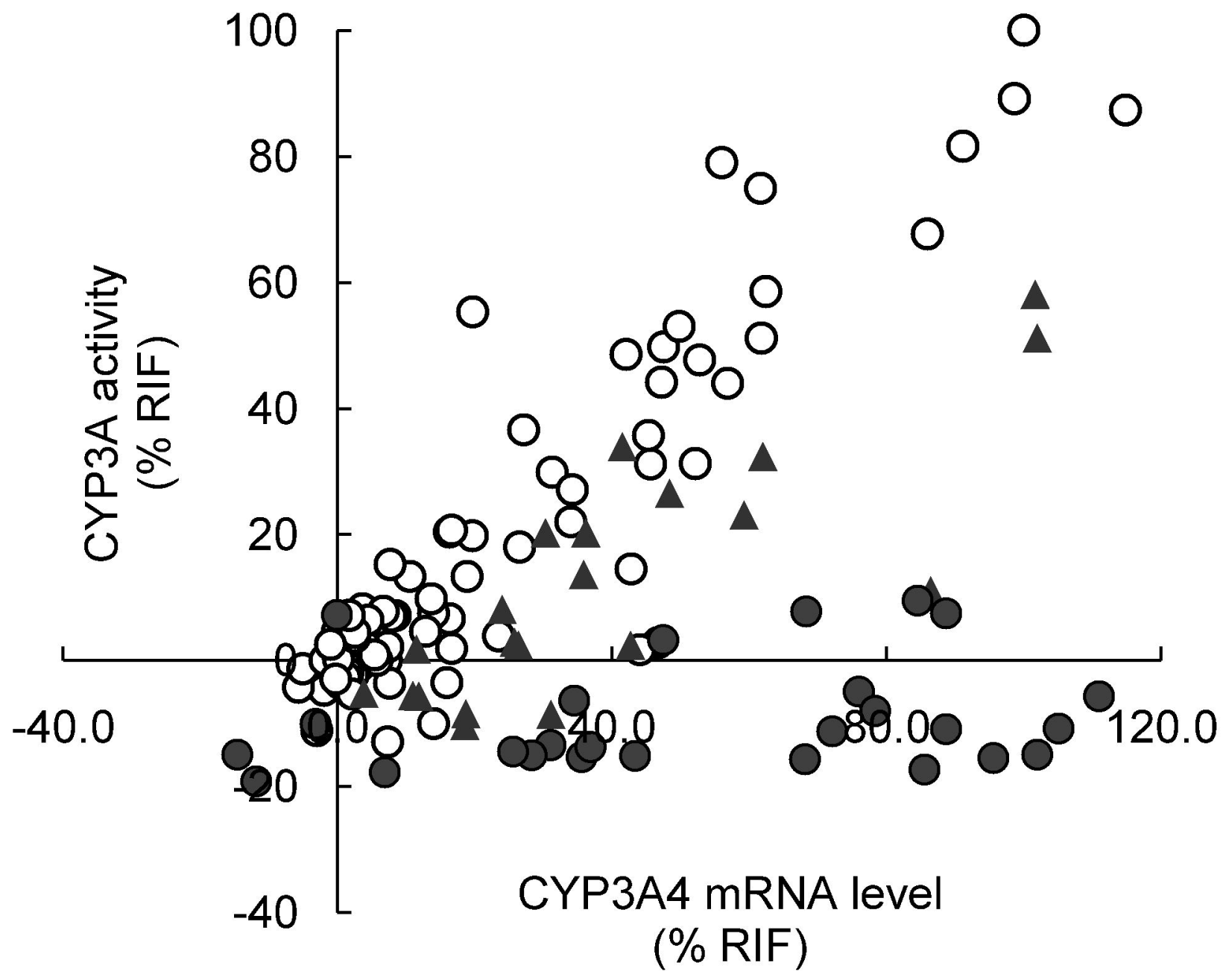

